\title{
Tratamiento en Lodo Activado del Lixiviado de un Relleno Sanitario
}

\author{
Carmen V. Droppelmann y Michael Oettinger \\ Universidad Andrés Bello, Facultad de Ecología y Recursos Naturales, República 440, $2^{\circ}$ piso, \\ Santiago-Chile (e-mail: vdroppelmann@unab.cl)
}

\begin{abstract}
Resumen
En este trabajo se estudia el comportamiento de un lodo activado en cuanto a su capacidad para remover la demanda biológica de oxígeno (DBO) y el nitrógeno amoniacal de un lixiviado previamente tratado en una laguna anaeróbica. El lixiviado pretratado presenta concentraciones de $\mathrm{DBO}_{5}$ y de $\mathrm{N}-\mathrm{NH}_{4}$ entre 1150 y $3017 \mathrm{mg} / \mathrm{l}$ y 1350 y $1750 \mathrm{mg} / \mathrm{L}$ respectivamente. La investigación se llevó a cabo en un reactor piloto de 30L, operando a tres factores de carga. Al mayor factor de carga aplicado $\left(0,073 \mathrm{~kg} \mathrm{DBO} / \mathrm{kg}\right.$ SSV día, $\left.0,07 \mathrm{~kg} \mathrm{~N}-\mathrm{NH}_{4} / \mathrm{SSV}\right)$, operando con un tiempo de residencia hidráulico de 10 días, se logran altas remociones de $\mathrm{DBO}_{5}\left(98 \%, 0,24 \mathrm{~kg} \mathrm{DBO} / \mathrm{m}^{3}\right)$ y de $\mathrm{N}-\mathrm{NH}_{4}$ $\left(99 \%, 0,15 \mathrm{~kg} \mathrm{~N}-\mathrm{NH}_{4} / \mathrm{m}^{3}\right.$ día). En estas condiciones de operación, las concentraciones de $\mathrm{DBO}_{5}(41$ $\mathrm{mg} / \mathrm{L})$, y de $\mathrm{N}-\mathrm{NH}_{4}(10 \mathrm{mg} / \mathrm{L})$ a la salida del lodo activado, cumplirían con holgura la norma chilena de emisión a alcantarillado.
\end{abstract}

Palabras clave: lixiviado, lodo activado, relleno sanitario, amonio, demanda biológica de oxígeno

\section{Treatment of Landfill Leachate using Activated Sludge}

\begin{abstract}
In this work the behavior of an activated sludge is studied according to its capacity to remove the biochemical oxygen demand (BOD) and ammonium nitrogen of a leachate previously treated in an anaerobic pond. The $\mathrm{BDO}_{5}$ and $\mathrm{N}-\mathrm{NH}_{4}$ concentrations in the pretreated leachate ranged from 1150 to $3017 \mathrm{mg} / \mathrm{L}$ and between 1350 to $1750 \mathrm{mg} / \mathrm{L}$ respectively. The study was carried out in a pilot scale reactor of $30 \mathrm{~L}$ operating at three load factors. At higher load factor $(0.073 \mathrm{~kg} \mathrm{BOD} / \mathrm{kg}$ SSV day, 0.07 $\left.\mathrm{kg} \mathrm{N}-\mathrm{NH}_{4} / \mathrm{SSV}\right)$, operating with a hydraulic residence time of 10 days, high $\mathrm{BOD}_{5}(98 \%, 0.24 \mathrm{~kg}$ $\mathrm{BOD}_{5} / \mathrm{m}^{3}$ day) and high $\mathrm{N}-\mathrm{NH}_{4}$ removals $\left(99 \%, 0.15 \mathrm{~kg} \mathrm{~N}-\mathrm{NH}_{4} / \mathrm{m}^{3}\right.$ day) were achieved. Under these operating conditions the effluent concentrations of $\mathrm{BOD}_{5}(41 \mathrm{mg} / \mathrm{L})$ and $\mathrm{N}-\mathrm{NH}_{4}(10 \mathrm{mg} / \mathrm{l})$ complies with the official Chilean sewage emission standards.
\end{abstract}

Keywords: leachate, activated sludge, landfill, ammonia, biochemical oxygen demand 


\section{INTRODUCCIÓN}

Los rellenos sanitarios, en la mayoría de los países, corresponden al destino final más común de los residuos sólidos domiciliarios (Renou et al., 2008; Lei et al., 2007; Moraes y Bertazzoli, 2005; Ding et al., 2001). Uno de los problemas ambientales más serios generados por los rellenos sanitarios corresponde a sus residuos líquidos, residuos conocidos como lixiviados (Primo et al., 2007; Karadag et al., 2007; Kurniawan et al., 2006).

Los lixiviados se generan a partir del agua que traen los residuos, como producto de la percolación del agua de lluvia a través del relleno y por los procesos bioquímicos al interior de las células (Renou et al., 2008). Los lixiviados pueden contener grandes cantidades de materia orgánica, nitrógeno amoniacal, metales pesados y sales (Uygur y Kargi, 2004; Renou et al., 2008; Primo et al., 2007). Las características químicas de los lixiviados varían de manera significativa en función de numerosos factores como son: el clima, la edad del relleno, la composición de la basura y la geología del terreno (Karadag et al, 2007; Cabeza et al., 2007; Peng et al., 2008).

Para tratar los lixiviados se utilizan muchos tipos de tratamiento y distintas combinaciones de estos (Trebouet et al., 2001; Ilhan et al., 2007; Uygur y Kargi, 2004; Kargi y Pamukoglu 2003). Debido a su fiabilidad, simplicidad y una conveniente relación costo beneficio, los tratamientos biológicos son comúnmente usados para remover la DBO de los lixiviados, en particular, si se trata de procesos biológicos aeróbico estos además, pueden nitrificar el nitrógeno amoniacal (Renou et al., 2008; Hoilijoki et al., 2000).

La constante de afinidad de Monod corresponde a la concentración de sustrato en la que la velocidad de crecimiento corresponde a un medio de la máxima. La constante de afinidad de Monod por el oxígeno de los microorganismos que remueve la DBO en forma aeróbica es pequeña (Robinson et al., 2004). Chuang et al. (1997), estudian el efecto de la concentración de oxígeno en la remoción de $\mathrm{DQO}$ en un lodo activado obteniendo la misma remoción a concentraciones de 2, 0,5 y 0,1 mg/L, similar resultado muestran Lau et al. (1984) operando un quimioestato en el que la concentración de oxígeno disuelto $(6,1,0,35$ y $0,09 \mathrm{mg} / \mathrm{L})$ no parece afectar la remoción de DQO.

La nitrificación demanda oxígeno y los microorganismos nitrificantes se caracterizan por ser muy sensibles a las condiciones ambientales, crecer lento y ser relativamente intolerantes a bajas concentraciones de oxígeno (Rittmann y McCarty, 2001). La nitrificación ocurre en dos etapas, en la primera el nitrógeno amoniacal es convertido en nitrito (nitritación) y en la segunda el nitrito es transformado en nitrato (nitratación) (Rittmann y McCarty, 2001).

Campos et al. (2007), trabajando en lodo activado muestran el efecto de la concentración de oxígeno disuelto en la nitrificación, cuando operan con concentraciones de oxígeno disuelto mayores a 1 $\mathrm{mg} / \mathrm{L}$ la nitrificación es total y no lo es a concentraciones de 0,4 y 0,6 mg/L, esto es coherente con lo reportado por Fan et al. (2000), quienes indican que la velocidad de nitrificación puede verse afectada con concentraciones de oxígeno disuelto menores a $1 \mathrm{mg} / \mathrm{L}$.

Satoh et al. (2003), al trabajar con flóculos de lodo activado, muestran como la velocidad de nitrificación (como producción de $\mathrm{NO}_{3}$ ) aumenta en la medida que aumenta la concentración de oxígeno en el seno del líquido, logrando la velocidad máxima a una concentración de oxígeno $40 \mu \mathrm{M}$ (1,3 $\mathrm{mg} / \mathrm{L})$. Jianlong y Ning (2004), verifican en lodo de reactor de membrana una menor velocidad máxima de oxidación del $\mathrm{N}-\mathrm{NH}_{4}$ (como remoción de $\left.\mathrm{N}-\mathrm{NH}_{4}\right)$ a 0,5 mg/L que a 1,5 mg/L.

Según diversos autores (Hunik et al., 1992; Nowak et al., 1995; Wiesmann, 1994; Guisasola et al., 2005) la constante de afinidad de Monod para oxígeno en la nitritación es menor que la constante de afinidad en la nitratación, esto implica que bajas concentraciones de oxígeno favorecen la acumulación de nitrito. Ruiz et al. (2003), operando un lodo activado a distintas concentraciones de oxígeno disuelto, muestran una acumulación de nitrito a concentraciones de oxígeno de 1,4 y 0,7 $\mathrm{mg} / \mathrm{L}$ y una disminución de la oxidación del $\mathrm{N}-\mathrm{NH}_{4}$ a 0,5 mg de oxígeno/L. Canziani et al. (2006), en un reactor de membrana, trabajando a concentraciones de oxígeno disuelto menores de $0,5 \mathrm{mg} / \mathrm{L}$, consiguen que ocurra nitritación y que se inhiba la nitratación. 
Es bien sabido que altas concentraciones de $\mathrm{N}-\mathrm{NH}_{4}$ inhiben la nitrificación (Li et al., 1999) y que las bacterias heterótrofas son menos sensibles a este compuesto (Lee et., 2000). Respecto a la inhibición de la remoción de DBO por nitrógeno amoniacal Li y Zhao (2001), trabajando durante 6 días con lodos activados operando en paralelo, muestran que la remoción de DQO en un lixiviado decrece en un $8 \%$ cuando la concentración de $\mathrm{N}-\mathrm{NH}_{4}$ aumenta dentro del reactor desde 0,19 a 143 $\mathrm{mg} / \mathrm{L}$.

Castens y Rozich (1986), estudian el efecto de la concentración de amonio en la velocidad específica de crecimiento de bacterias nitrificantes, su estudio lo realizan en reactores batch con concentraciones iniciales de nitrógeno amoniacal entre 5 y $60 \mathrm{mg} / \mathrm{L}$, de acuerdo a sus resultados la inhibición ocurriría cuando la concentración de nitrógeno amoniacal supera un valor que se encuentra entre 15 y $20 \mathrm{mg} / \mathrm{L}$. De acuerdo a los estudios realizados por Buday et al. (1999), la inhibición se lleva a cabo por la molécula no ionizada y la nitratación es más sensible a la inhibición que la nitritación. Carrera et al. (2003), trabajan con un RIL en un lodo activado y cuando la concentración de amonio llega dentro del reactor a $500 \mathrm{mg} / \mathrm{L} \quad\left(7,7 \mathrm{mg} \mathrm{N}-\mathrm{NH}_{3} / \mathrm{L}\right)$ la velocidad de nitrificación se ve disminuida de 0,15 a $0,10 \mathrm{~g} \mathrm{~N}-\mathrm{NH}_{4} / \mathrm{g}$ SSV día.

Hoilijoki et al. (2000), estudian la nitrificación a bajas temperaturas de un lixiviado de relleno sanitario pretratado anaeróbicamente en un reactor de lecho fluidizado con flujo ascendente. El lixiviado pretratado posee concentraciones de $\mathrm{DBO}_{7}$ y de $\mathrm{N}$-amoniacal de $25-170 \mathrm{mg} / \mathrm{L}$ y de $53-270 \mathrm{mg} / \mathrm{L}$ respectivamente. La nitrificación la llevan a cabo en un lodo activado que opera con un tiempo de de residencia hidráulico de 2,7 días con cargas de $0,05 \mathrm{~kg} \mathrm{~N}-\mathrm{NH}_{4} / \mathrm{m}^{3}$ día y de $0,027 \mathrm{~g} \mathrm{~N}-\mathrm{NH}_{4} / \mathrm{g} \mathrm{SSVLM}$ día, logrando a $10^{\circ} \mathrm{C}$ un $100 \%$ de nitrificación y una $\mathrm{DBO}_{7}$ remanente menor a $7 \mathrm{mg} / \mathrm{L}$. Ağdağ y Sponza (2005), tratan en un reactor aeróbico agitado de mezcla completa un lixiviado pretratado en dos reactores anaeróbicos en serie del tipo lecho fluidizado con flujo ascendente. Operando con una carga (calculada) de $0,13 \mathrm{~kg} \mathrm{~N}-\mathrm{NH}_{4} / \mathrm{m}^{3}$ día (465 mg N-NH$/ \mathrm{L}, 320 \mathrm{mg} \mathrm{DBO} / \mathrm{L}$ y un tiempo de residencia hidráulico de 4,5 días) logran una remoción de $\mathrm{N}^{-\mathrm{NH}_{4}}$ sobre el $99 \%$.

En este trabajo se estudia el comportamiento de un lodo activado en cuanto a su capacidad para remover DBO y nitrógeno amoniacal $\left(\mathrm{N}-\mathrm{NH}_{4}\right)$ de un lixiviado previamente tratado en una laguna anaeróbica.

\section{MATERIALES Y MÉTODOS}

La investigación se llevó a cabo utilizando lixiviado generado en el relleno del consorcio Kiasa De Marco, Relleno Sanitario Loma Los Colorados $\left(33^{\circ} \mathrm{S}, 70^{\circ} \mathrm{O}\right)$, relleno que comenzó su operación hace 10 años. El lixiviado es pretratado, en el mismo recinto del relleno, por medio de lagunas anaeróbicas con un tiempo de residencia hidráulico mayor a 45 días.

Las experiencias se llevaron a cabo en un lodo activado piloto (PIGNAT, modelo TAE/1000), operando con $30 \mathrm{~L}$ de licor mezclado, aireado mediante difusores, alimentado mediante bomba peristáltica, con reciclo de lodo mediante bomba de membrana y a temperatura ambiente. El tanque aireado es sembrado con licor mezclado del lodo activado que trata los lixiviados en el relleno sanitario. El reactor se operó a tres factores de cargas distintas, dando tiempo al que el sistema se estabilice entre uno y otro factor de carga.

La $\mathrm{DBO}_{5}$ se determinó semanalmente mediante medición manométrica, utilizando el sistema Velp BMS 6 en la incubadora Velp FOC 225. Las concentraciones de $\mathrm{P}_{-} \mathrm{PO}_{4}, \mathrm{~N}-\mathrm{NH}_{4}$, nitrógeno total, nitrito y nitrato se determinaron quincenalmente usando el espectrofotómetro multiparamétrico $\mathrm{HACH}$ de acuerdo las instrucciones del fabricante. Los sólidos suspendidos totales y sólidos suspendidos volátiles se determinaron quincenalmente de acuerdo a los métodos estándares 2540 D y 2540 (APHA, 1995). La concentración de oxígeno disuelto se determina semanalmente utilizando un oxímetro marca WTW, modelo OXI 330. En general los análisis se realizan sin repeticiones a excepción de la DBO que se determina por triplicado.

La comparación de los datos obtenidos, para cada uno de los factores de carga, se realiza por comparación múltiple de media (Fisher, $\mathrm{p}_{0,05}$ ), previa verificación de normalidad (Kurtosis). 


\section{RESULTADOS Y DISCUSIÓN}

En la figura 1 se presentan las concentraciones de $\mathrm{DBO}_{5}$, nitrógeno total y $\mathrm{P}_{-} \mathrm{PO}_{4}$ a la entrada del reactor, de acuerdo a estas concentraciones y considerando la relación $\mathrm{DBO}_{5}: \mathrm{N}: \mathrm{P}=100: 5: 1$ (Winkler, 1999) el sistema no presenta requerimientos adicionales de nitrógeno y/o fósforo.

En las figuras 2 y 3 pueden verse los factores de carga (F/M) y tiempo de residencia hidráulico (TRH) obtenidos durante el transcurso de la experiencia, las líneas verticales dividen el gráfico en las fases I, II y III las que presentan F/M promedios de 0,014, 0,041 y 0,073 kg DBO $/ \mathrm{kg}$ SSV día y TRH promedios de 50, 21 y 10 días respectivamente.

En la figura 4, puede observarse la concentración de sólidos suspendidos totales y sólidos suspendidos volátiles en el licor mezclado. El porcentaje promedio de sólidos suspendidos volátiles respecto al a los sólidos suspendidos totales, en cada una de las fases corresponde a 56, 62 y $65 \%$, siendo estos estadísticamente distintos entre si. Como es de esperarse, el porcentaje de volátiles aumenta al disminuir el tiempo de residencia hidráulico, ya que esto implica un menos tiempo de residencia del lodo (Winkler, 1999).

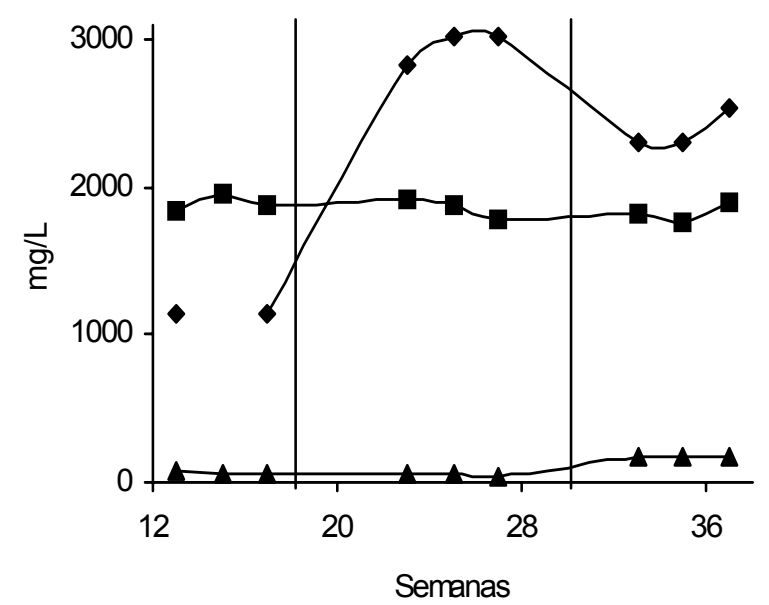

Fig. 1: Concentración de $\mathrm{DBO}_{5}(\triangleleft)$, nitrógeno total (-) y $\mathrm{P}_{-} \mathrm{PO}_{4}(\boldsymbol{\Delta})$ a la entrada del lodo activado.

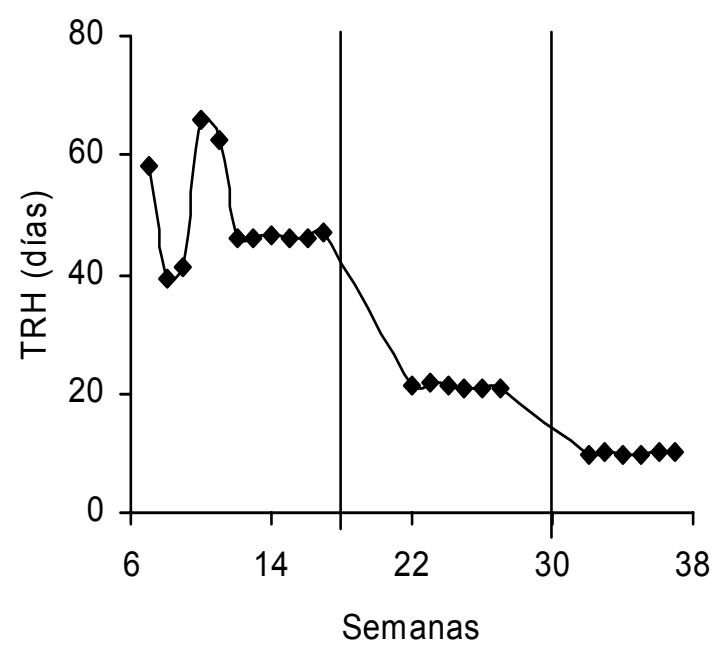

Fig. 3: Tiempo de residencia hidráulico.

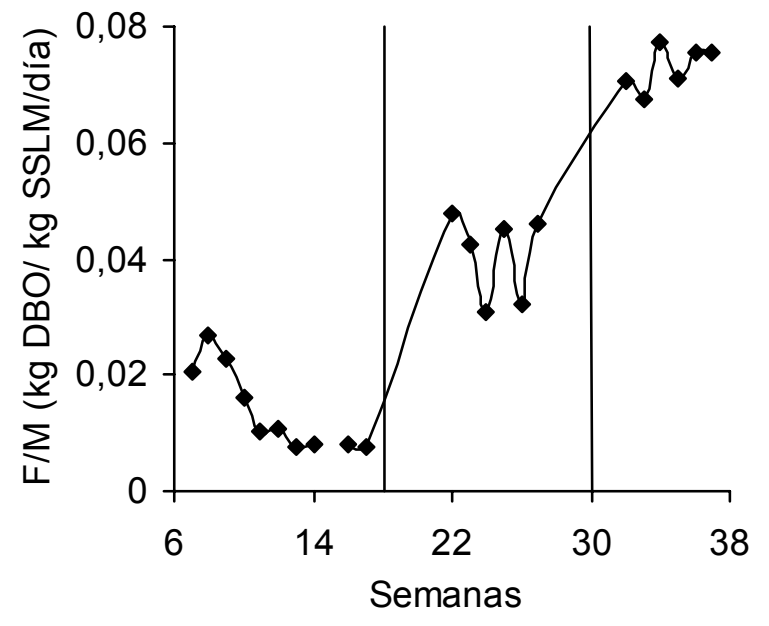

Fig. 2: Factor de carga (F/M).

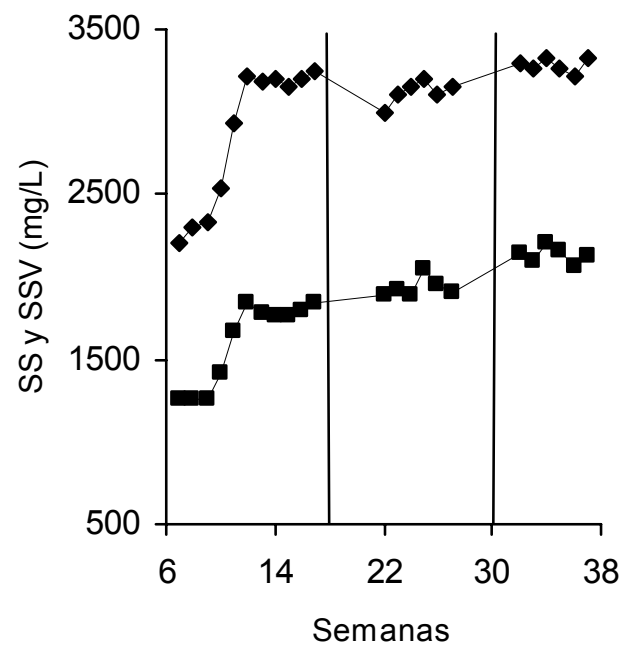

Fig. 4: Sólidos suspendidos totales $(\bullet)$ y volátiles (घ) del licor mezclado. 


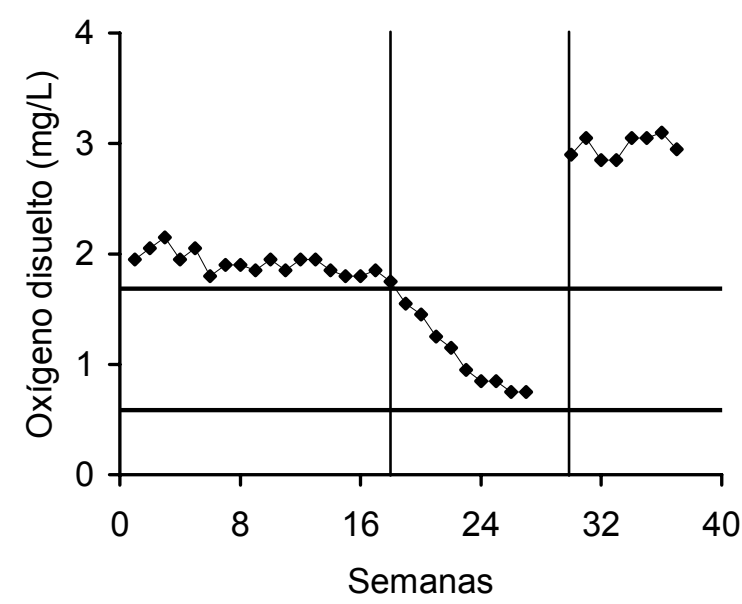

Fig. 5: Concentración de oxígeno disuelto en el licor mezclado.

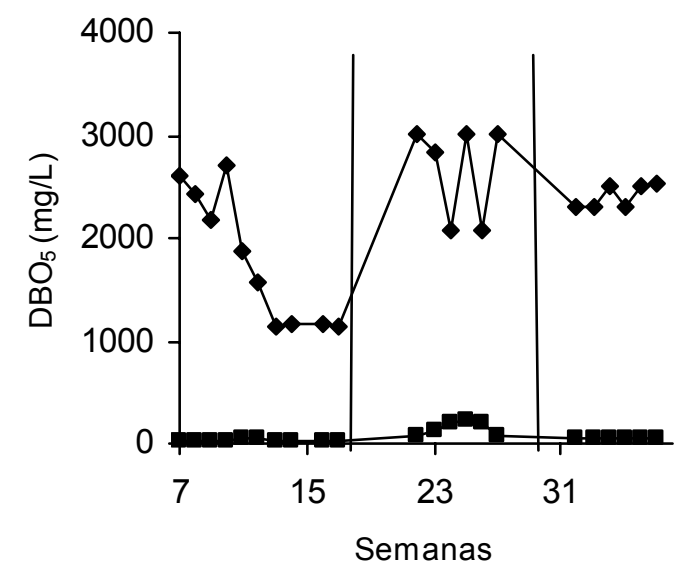

Fig. 6: $\mathrm{DBO}_{5}$ a la entrada $(\bullet)$ y salida $(\mathbf{\square})$ del lodo activado.

Las remociones de $\mathrm{DBO}_{5}$ (figura 6) son de 99, 94 y $98 \%$ para las fases I, II y III respectivamente. La mayor velocidad promedio de remoción se logra en la fase III y es de $0,24 \mathrm{~kg} \mathrm{DBO} / \mathrm{m}^{3}$ día. El menor porcentaje de remoción (estadísticamente significativa) obtenida en la fase II es coherente con la relativamente baja concentración de oxígeno que impide que la nitrificación se lleve a cabo adecuadamente, presentándose un exceso de $\mathrm{N}-\mathrm{NH}_{4}$ en el tanque aireado (figura 8), el que a su vez presentaría un efecto inhibitorio sobre las bacterias heterótrofas encargadas de remover la DBO (Metcalf y Eddy, 1991; Li y Zhao, 2001).

Junto a una menos remoción de $\mathrm{N}-\mathrm{NH}_{4}$ en la fase II se observa una acumulación promedio de 200 $\mathrm{mg} / \mathrm{L}$ de nitrito, proceso observado frecuentemente en la nitrificación de lixiviados y atribuible entre otras causas a bajas concentraciones de oxígeno disuelto (Hunik et al., 1992; Wiesmann 1994; Nowak et al. 1995; Ruiz et al., 2003; Guisasola et al., 2005; Canziani et al., 2006).

Las concentraciones promedios de $\mathrm{DBO}_{5}$ a la salida del lodo activado resultan de 24,152 y $41 \mathrm{mg} / \mathrm{L}$ para cada fase, estas concentraciones cumplen con la norma de emisión a alcantarillado (D.S. 609/1998). La concentración de la fase I está de acorde con la norma chilena de emisión a cursos de aguas superficiales (D.S. 90/ 2000).

Las concentraciones promedios de $\mathrm{N}-\mathrm{NH}_{4}$ a la salida del reactor para cada una de las fases son de 3,275 y $10 \mathrm{mg} / \mathrm{L}$ (99,7 82,0 y 99,3\% de remoción estadísticamente diferentes entre si) (figura 7 ). Las concentraciones de la fase I y III según la literatura (Castens y Rozich, 1986; Carrera et al., 2003) no son lo suficientemente altas como no ejercerían inhibición por sustrato y según la normas de emisión chilenas serían aptas para descargar a alcantarillado (D.S. 609/1998).

La mayor velocidad promedio de remoción de $\mathrm{N}-\mathrm{NH}_{4}$ es $0,15 \mathrm{~kg} \mathrm{~N}-\mathrm{NH}_{4} / \mathrm{m}^{3}$ día y se logra en la fase III en la que se aplican $0,07 \mathrm{~kg}$ de N-NH${ }_{4} / \mathrm{kg}$ SSV día. Estos valores son bastante mayores a los 0,05 $\mathrm{kg} \mathrm{N}-\mathrm{NH}_{4} / \mathrm{m}^{3}$ día logrados de Hoilijoki et al., 2000 y similares a los $0,13 \mathrm{~kg} \mathrm{~N}-\mathrm{NH}_{4} / \mathrm{m}^{3}$ día obtenidos por Ağdağ y Sponza (2005). Es interesante señalar que la velocidad promedio de remoción en la fase III, a pesar de la complejidad del residuo, es similar a los $0,2 \mathrm{~kg} \mathrm{~N}-\mathrm{NH}_{4} / \mathrm{m}^{3}$ día típico de lodos activados que tratan aguas servidas (Campos et al., 1999).

La mayor generación de nitrato, $0,08 \mathrm{~kg} / \mathrm{m}^{3}$ día, ocurre en la fase III. A pesar de estar en un sistema de tratamiento aeróbico, ocurre una remoción de nitrógeno (figura 8). Esto se ha observado en otras plantas aeróbicas y se explica por la ausencia de oxígeno molecular al interior del flóculo lo que 
permite la ocurrencia de desnitrificación y de oxidación anaeróbica del amonio (Schramm et al., 1999; Satoh et al., 2003; Deber et al., 1997).

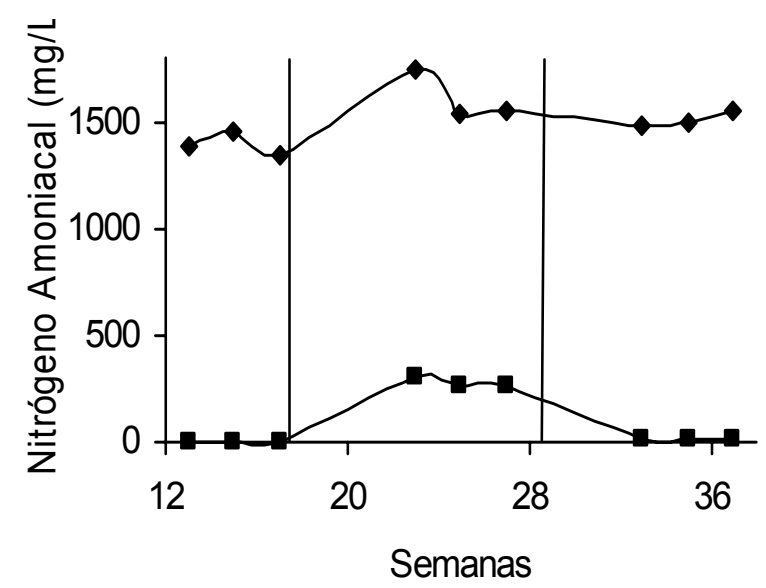

Fig. 7: Concentración de nitrógeno amoniacal a la entrada $(\diamond)$ y salida ( $\mathbf{\square})$ del lodo activado.

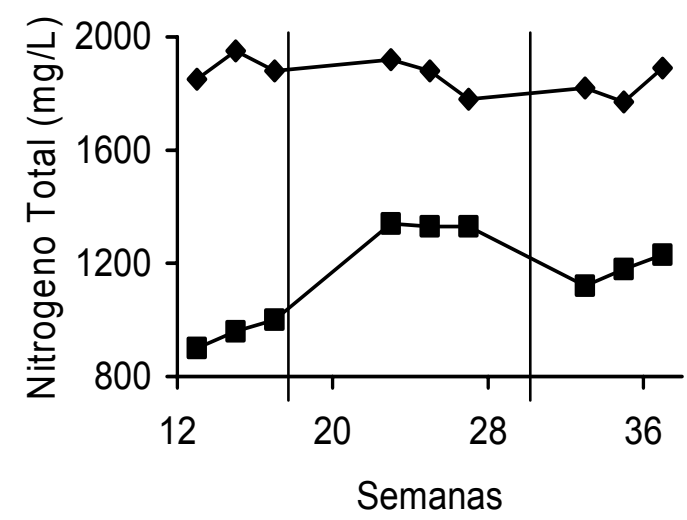

Fig.8: Concentración de nitrógeno total a la entrada $(\diamond)$ y salida ( $\mathbf{\square})$ del reactor

En la figura 9 pueden verse las concentraciones de entrada y salida de $\mathrm{P}_{-} \mathrm{PO}_{4}$. En la fase III a pesar de que aumenta la concentración de entrada de $\mathrm{P}_{-} \mathrm{PO}_{4}$ el sistema es capaz de abatirlo. Respecto a esto, hay que considerar que el menor $\mathrm{TRH}$, condición en que la mayor generación de lodo, favorece la remoción de $\mathrm{P}_{-} \mathrm{PO}_{4}$ y que estamos frente al efecto de la dilución, efecto que podría ser insuficiente si la concentración de entrada de fósforo se mantiene alta en el tiempo.

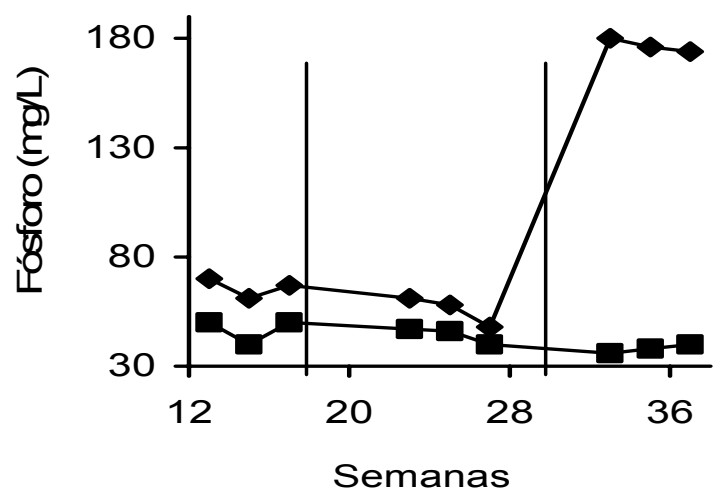

Fig. 9: Concentración de fósforo a la entrada $(\diamond)$ y salida (ロ) del lodo activado.

\section{CONCLUSIONES}

De acuerdo a los resultados obtenidos, es posible lograr altas remociones de $\mathrm{DBO}_{5}(98 \%, 0,24 \mathrm{~kg}$ $\mathrm{DBO}_{5} / \mathrm{m}^{3}$ día) y de $\mathrm{N}-\mathrm{NH}_{4}\left(99 \%, 0,15 \mathrm{~kg} \mathrm{~N}-\mathrm{NH}_{4} / \mathrm{m}^{3}\right.$ día) cuando al lodo activado se aplican $0,073 \mathrm{~kg}$ $\mathrm{DBO}_{5} / \mathrm{kg}$ SSV día (TRH 10 días) y $0,07 \mathrm{~kg} \mathrm{~N}-\mathrm{NH}_{4} / \mathrm{SSV}$ día. A estas condiciones de operación, las concentraciones de $\mathrm{DBO}_{5}(41 \mathrm{mg} / \mathrm{L})$, y de $\mathrm{N}-\mathrm{NH}_{4}(10 \mathrm{mg} / \mathrm{L})$ a la salida del lodo activado, cumplirían con holgura la norma chilena de emisión a alcantarillado (D.S. N609/1998). 


\section{REFERENCIAS}

Ağdağ, O.N. y D T. Sponza; Anaerobic/Aerobic Treatment of Municipal landfill Leachate in Sequential Two-Stage Up-Flow Anaerobic Sludge Blanket Reactor (UASB)/Completely Stirred Tank Reactor (CSTR) Systems, Process Biochemistry: 40(2), 895-902 (2005).

APHA (American Public Health Association); Standard Methods for the Examination of Water and Wastewater, 20 Ed., Washington, USA (1995).

Buday, J., M. Drtil, M. Hutňan y J. Derco; Substrate and Product Inhibition of Nitrification, Chemical Papers: 53(6), 379-383 (1999).

Cabeza, A., A. Urtiaga, M. J. Rivero e I. Ortiz; Ammonium Removal from Landfill Leachate by Anodic Oxidation, Journal of Hazardous Materials: 144 (3), 715-719 (2007).

Campos, J.L., J.M. Garrido-Fernández, R. Méndez y J.M. Lema; Nitrification at High Ammonia Loading Rates in an Activated Sludge Unit, Bioresource Technology: 68(2), 141-148 (1999).

Campos, J.L., J.M. Garrido, A. Mosquera-Corral y R. Mendez; Stability of a nitrifying Activated Sludge Reactor, Biochemical Engineering Journal: 35(1), 87-92 (2007).

Canziani, R. y otros cinco autores; Effect of Oxygen Concentration on Biological Nitrification and Microbial Kinetics in a Cross- Flow Membrane Bioreactor (MBR) and Moving-Bed Biofilm Reactor (MBBR) Treating Old Landfill Leachate, Journal of Membrane Science: 286(1-2), 202-212

Carrera J., J.A Baeza, T. Vicente y J. Lafuente; Biological Nitrogen Removal of High-Strength Ammonium Industrial Wastewater with Two-Sludge System, Water Research 37(17), 4211-4221 (2003).

Castens, D.J. y A.F. Rozich; Analysis of Batch Nitrification using Substrate Inhibition Kinetics, Biotechnology and Bioengineering: 28(3), 461-465 (1986).

Chuang, S.H., C.F. Ouyang, H.C. Yuang y S.J. You; Effects of SRT and DO on Nutrient Removal in a combined AS-Biofilm Process, Water Science Technology: 36(12), 19-27 (1997).

Debeer, D., A. Schramm, C.M. Santegoeds y M. Kühl; A Nitrite Microsensor for Profiling Environmental Biofilms, Applied Environmental Microbiology: 63(3), 973-977 (1997).

Ding, A., Z. Zhang, J. Fu y L. Cheng; Biological Control of leachate from municipal landfills, Chemosphere: 44(1), 1-8 (2001).

D.S. N90/2000: Ministerio Secretaria General de la Presidencia (Chile), Norma de Emisión para la Regulación de Contaminantes Asociados a las Descargas de Residuos Líquidos a Aguas Marinas y Continentales Superficiales, Chile (2000).

D.S. N609/1998: Ministério de Obras Públicas (Chile), Norma de Emisión para la Regulación de Contaminantes Asociados a las Descargas de Residuos Industriales Líquidos a Sistemas de Alcantarillado, Chile (1998).

Fan, X.J. y otros cinco autores; Nitrification in a Membrane Bioreactor (MBR) for Wastewater Treatment, Water Science Technology: 24(3-4), 289-294 (2000).

Guisasola, A. y otros cuatro autores; Respirometric Estimation of the Oxygen Affinity Constant for Biological Ammonium and Nitrite Oxidation, Journal of Chemical Technology and Biotechnology: 80(4), 388-396 (2005)

Hoilijoki, T.H., R.H. Kettunen y J.A. Rintala; Nitrification of Anaerobically Pretreated Municipal Landfill Leachate at Low Temperature, Water Research: 34(5), 1435-1446 (2000). 
Hunik, J.H., J. Tramper y R.H. Wijffels; A Strategy to Scale Up Nitrification Processes with Inmobilized Cells of Nitrosomonas europaea and Nitrobacter agilis, Bioprocess Engineering 11(2), 73-82 (1992)

Ilhan, F., U. Kurt, O. Apaydin y M.T. Gonollu; Treatment of Leachate by Electrocoagulation using Aluminum and Iron Electrodes, Journal of Hazardous Materials (2007), doi:10.10106/j.jhazmat. 2007.10.035.

Jianlong, W. e Y. Ning; Partial Nitrification under Limited Dissolved Oxygen Conditions, Process Biochemistry: 39(1), 1223-1229 (2004).

Karadag, D. y otros cinco autores; Ammonium removal from Sanitary Landfill Leachate using Natural Gördes Clinoptilolite, Journal of Hazardous Materials (2007), doi:10.10106/j.jhazmat.2007.08.019.

Kargi, F. y M.Y. Pamukoglu; Powdered Activated Carbon Added Biological Treatment of Pre-Treated Landfill Leachate in a Fed-Batch Reactor, Biotechnology Letters: 25(9), 695-699 (2003).

Kurniawan, T.A., W. Lo y G. Chan; Physico-Chemical Treatments for Removal of Recalcitrant Contaminants from Landfill Leachate, Journal of Hazardous Materials: 129(1-3), 80-100 (2006).

Lau, A.O., P.F. Sttrom y D. Jenkis; Growth Kinetics of Sphaerotilus natans and a flocforming in pure and dual continuous culture, Journal of the Water Pollution Control Federation: 56(1), 41-51 (1984).

Lee, S.M., J.Y. Jung e Y.C. Chung; Measurement of Ammonia Inhibition of Microbial Activity in Biological Wastewater Treatment Process using the Hydrogenase Assay, Biotechnology Letters, 22(12);991-994 (2000).

Lei, Y., Z. Shen, R. Huang y W. Wang; Treatment of landfill leachate by combined aged-refuse bioreactor and electro-oxidation, Water Research: 41(11), 2417-2426 (2007).

Li, X.Z. y Q.L. Zhao; Efficiency of Biological Treatment Affected by High Strength of AmmoniumNitrogen in Leachate and Chemical Precipitation of Ammonium-Nitrogen as Pretreatment, Chemosphere: 44(1), 37-43 (2001).

Li, X.Z., Q.L. Zhao y X.D. Hao; Ammonium Removal from Leachate by Chemical Precipitation, Waste Management: 19(6), 409-415 (1999).

Metcalf y Eddy Inc.; Wastewater Engineering, Treatment, Disposal, and Reuse, third edition, McGraw-Hill, Inc., New York (1991).

Moraes, P.B. y R. Bertazzoli; Electrodegradation of landfill leachate in a flow electrochemical reactor. Chemosphere: 58(1), 41-46 (2005).

Nowak, O., K. Svardal y P. Schweighofer; The Dynamic Behavior of the Nitrifying Activated Sludge Systems Influenced by Inhibiting Wastewater Compounds, Water Science technology: 31(2), 115-124 (1995).

Peng, Y. y otros cinco autores; Organic Removal by Denitritation and Methanogenesis and Nitrogen Removal by Nitritation from Landfill Leachate, Water Research: 42(4-5), 883-892 (2008).

Primo, O., M.J. Rivero y I. Ortiz; Photo-Fenton Process as an Efficient Alternative to the Treatment of Landfill Leachates, Journal of Hazardous Materials (2007), doi:10.10.106/j.jhazmat.2007.09.053.

Renou, S., y otros cuatro autores; Landfill Leachate Treatment : Review and Opportunity, Journal of Hazardous Materials: 150(3), 468-493 (2008).

Rittmann, B.E. y P.L. McCarty; Biotecnología del Medio Ambiente, Principios y Aplicaciones, 461-487, McGrawHill, Madrid, España (2001). 
Robinson, K.G., G.S. Sayler y J.R. Janalyn; Used of Novel Techniques to Quantify Phenotypes in Biological Treatment Process, 2.11-2.12.IWA Publishing, Orlando, USA (2004).

Ruiz, G., D. Jeison y R. Chamy; Nitrification with High Nitrite Accumulation for the Treatment of Wastewater with High Ammonia Concentration, Water Research 37(6), 1371-1377 (2003).

Satoh, H., Y. Nakamura, H. Ono y S. Okabe; Effect of Oxygen Concentration on Nitrification and Denitrification in Single Activated Sludge Flocs, Biotechnology and Bioengineering: 83(5), 604-607 (2003).

Schramm, A. y otros ocho autores; On the Occurrence of Anoxic Microniches, Denitrification, and Sulfate Reduction in Aerated Activated Sludge, Applied Environmental Microbiology: 65(9), 41894196 (1999).

Trebouet, D., J.P. Schlumpf, J. Jaquen y F. Quemeneur; Stabilized Landfill Leachate Treatment by Combined Physcochemical-Nanofiltration Processes, Water Research: 35(12), 2935-2942 (2001).

Uygur, A. y Kargi, F.; Biological Nutrient Removal from Pre-Treated Landfill Leachate in a Sequencing Batch Reactor, Journal of Environmental Management: 71(1), 9-14 (2004).

Winkler, M.A.; Tratamiento Biológico de Aguas de Desecho, 87-93. Limusa, Madrid, España (1999).

Wiesmann, U.; Biological Nitrogen Removal from Wastewater, In Advances in Biochemical Engineering by A. Fletcher, pp 113-154 Springer-Verlag, Berlin, Alemania (1994). 
